\title{
Estudio para instalar una planta procesadora de puré instantáneo de zapallo macre
}

\author{
María Claudia Merino Castañeda* \\ Geraldine Mercedes Otiniano Osorio* \\ Universidad de Lima. Lima, Perú \\ Recibido: 27 de abril de 2014 / Aprobado: 27 de mayo de 2014
}

\begin{abstract}
RESUMEN: El zapallo macre es una hortaliza que se caracteriza por su gran tamaño y por su alto contenido de betacaroteno y vitamina A. Es considerado uno de los alimentos más conocidos por las amas de casa peruanas, pues es el ingrediente principal de varios platos típicos. Y es que el zapallo, a pesar de encontrarse en abundancia, no ha sido verdaderamente industrializado, dándole un valor agregado. Este estudio plantea la fabricación de puré instantáneo a partir de este insumo; se definirá su mercado meta, la tecnología necesaria para su desarrollo y una evaluación económicofinanciera para asegurar la rentabilidad del proyecto.
\end{abstract}

Palabras clave: zapallo macre / hortalizas / puré instantáneo / betacaroteno / vitamina A

\section{Study for to install a production plant of instant puree of macre pumpkin}

AвstRAct: The macre pumpkin is a vegetable characterized by its huge size and its high content of beta-carotene and vitamin A. It is considered one of known foods by peruvian housewives because it is the main ingredient of many traditional dishes. What is more, the pumpkin, though its abundance, it has not been really industrialized, in other words, it has not been giving added value. This study proposes the manufacture of instant puree from this ingredient in which its target market will be defined, the technology required for development and economic-financial evaluation to ensure the profitability of the project.

Key words: macre pumpkin / vegetables / instant puree / beta-carotene / vitamin $A$

* Correos electrónicos: mclaudia9999@hotmail.com, geraldine.0801@gmail.com. 


\section{ANTECEDENTES Y MARCO TEÓRICO}

En el país existe una costumbre arraigada de consumo de zapallo, que data de la época preincaica, entre 4200 y 2500 a. C., según los restos hallados en el departamento de Lima. La variedad más común de zapallo en el Perú es la denominada macre (nombre científico Cucúrbita máxima Duch). Esta hortaliza se cultiva tanto en la costa como en la sierra durante todo el año y se distingue por su típico color amarillo y gran tamaño. Se vende cuando está fresca, ya sea entera o en trozos, tanto en los mercados como en los supermercados.

\subsection{Propiedades y usos}

El zapallo macre se caracteriza por tener propiedades nutricionales, entre las que se encuentran:

- Es bajo en calorías, por su escaso contenido de grasas y carbohidratos.

- Tiene propiedades antioxidantes por su alto contenido de betacaroteno y vitamina A.

- Posee considerable contenido en minerales, como potasio, fósforo y calcio.

Se utiliza como principal insumo en la preparación de:

- Papillas para bebés.

- Diversos platos, como locro, sopa, puré, entre otros.

- Postres: queques, mazamorras, helados.

Se puede resaltar su bajo contenido de kilocalorías ( $25 \mathrm{kcal})$ en comparación con 100 gramos de papa, que tienen 76 kcal. También se observa su alto contenido en carotenos.

En la tabla 1 se muestra el contenido nutricional de 100 gramos de zapallo macre. 
Tabla 1

Composición de 100 gramos de zapallo macre

\begin{tabular}{|c|c|}
\hline Componente & $\begin{array}{l}\text { Contenido por } \\
\text { cada } 100 \text { gramos }\end{array}$ \\
\hline Agua (g) & 91 \\
\hline Energía (kcal) & 25 \\
\hline Proteínas (g) & 1.1 \\
\hline Hidratos de carbono (g) & 4.6 \\
\hline Lípidos (g) & 0.13 \\
\hline \multicolumn{2}{|l|}{ Fibra } \\
\hline Fibra total $(\mathrm{g})$ & 2.2 \\
\hline Soluble (g) & 0.9 \\
\hline Insoluble (g) & 1.3 \\
\hline \multicolumn{2}{|l|}{ Vitaminas } \\
\hline Vitamina A $(\mu \mathrm{g})$ & 128 \\
\hline Carotenos totales $(\mu \mathrm{g})$ & 949 \\
\hline Alfa caroteno $(\mu \mathrm{g})$ & 247 \\
\hline Beta caroteno $(\mu \mathrm{g})$ & 582 \\
\hline Criptoxantina $(\mu \mathrm{g})$ & 120 \\
\hline Vitamina $E(\mu \mathrm{g})$ & 1100 \\
\hline Vitamina B1 $(\mu \mathrm{g})$ & 50 \\
\hline Vitamina B2 $(\mu \mathrm{g})$ & 70 \\
\hline Niacina $(\mu \mathrm{g})$ & 500 \\
\hline Vitamina B6 $(\mu \mathrm{g})$ & 110 \\
\hline Folatos $(\mu \mathrm{g})$ & 36 \\
\hline Vitamina C $(\mu \mathrm{g})$ & 1200 \\
\hline \multicolumn{2}{|l|}{ Minerales } \\
\hline Calcio (mg) & 22 \\
\hline Hierro (mg) & 0.8 \\
\hline Fósforo (mg) & 44 \\
\hline Magnesio (mg) & 8 \\
\hline Zinc (mg) & 0.2 \\
\hline Sodio (mg) & 3.1 \\
\hline Potasio (mg) & 304 \\
\hline
\end{tabular}

Fuente: Moreiras et al., 2001. 


\section{ESTUDIO DE MERCADO}

\subsection{Definición del producto}

El puré instantáneo a partir de zapallo macre es un producto innovador, de sabor agradable, reducido en grasas, rico en betacaroteno y vitaminas. Su insumo principal, el zapallo, se deshidratará y venderá en forma de polvo con otros agregados para su mejor preservación y sabor. Al agregarle agua caliente al producto, se rehidratará volviéndose puré.

Se venderá en sobres de 125 gramos, que rinden aproximadamente 4 porciones. El precio unitario se ha definido en 2.60 soles, que es el precio promedio del principal producto sustituto, el puré de papa instantáneo. En la figura 1 se observa el diseño del empaque.

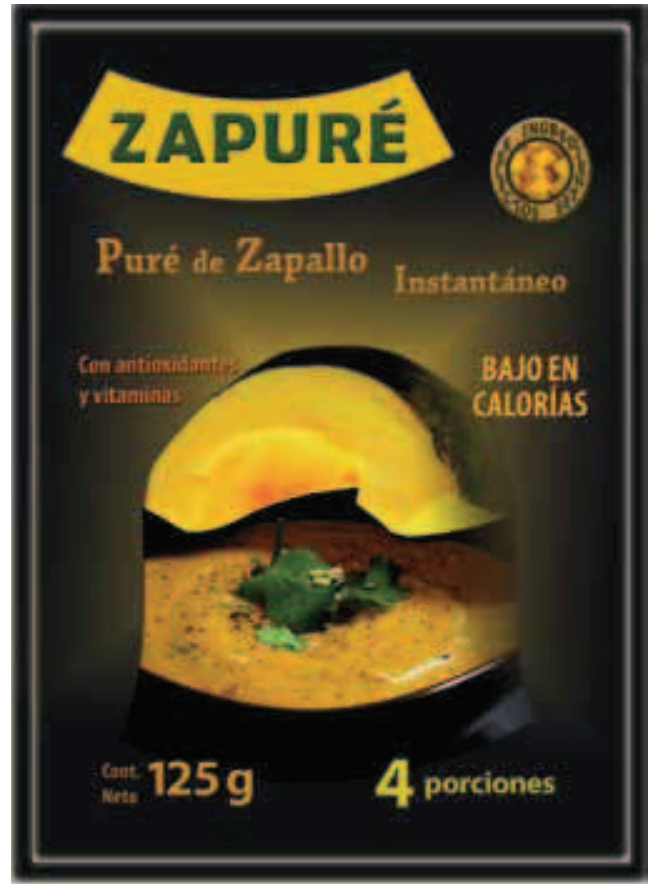

Elaboración propia. 


\subsection{Segmentación de mercado y determinación de mercado meta}

Las principales variables que deben analizarse para poder conseguir una segmentación adecuada de mercado son:

- Segmentación geográfica. Al ser un nuevo producto el primer objetivo es hacer que este sea conocido, y para esto lo más conveniente es buscar el mercado con mayor población posible. Hoy en día Lima metropolitana tiene una población aproximada de 9 millones de habitantes; es la zona más poblada del Perú.

- Segmentación psicográfica. El producto puré instantáneo de zapallo se enfocará a los niveles socioeconómicos (NSE) A y B, por ser estos los que poseen patrones de consumo marcadamente más favorables hacia productos de reconstitución instantánea como el puré de papa, tal como se observa en la tabla 2. La penetración de mercado es la más alta en estos sectores.

Tabla 2

Penetración de mercado del puré de papa instantáneo en Lima según NSE

\begin{tabular}{cccccc}
\hline Año & NSE A\% & NSE B $\%$ & NSE C $\%$ & NSE D $\%$ & NSE E\% \\
\hline 2007 & 17 & 13 & 4 & 3 & 4 \\
$2008^{*}$ & - & - & - & - & - \\
$2009^{*}$ & - & - & - & - & - \\
2010 & 20 & 8 & 4 & 0 & 0 \\
2011 & 21 & 16 & 6 & 4 & 4 \\
\hline
\end{tabular}

* No hay información

Fuente: Ipsos Apoyo, 2011.

Elaboración propia.

- Segmentación demográfica. El producto en cuestión está orientado principalmente a las amas de casa de los NSE A y B, que tienen un una edad promedio de 43 años, el $50 \%$ está casada y más del $80 \%$ tiene hijos. Ellas son las que comprarán el producto para consumo en su hogar; se debe tener en vuenta que el ama de casa es "[...] la persona encargada de organizar las labores domésticas, así como de tomar las decisiones finales acerca de compra de productos para el hogar, por lo que su papel es de gran importancia dentro del mismo" 
(Ipsos Apoyo, 2010). La mayor parte de las mujeres de este estrato están incorporadas a la vida laboral y trata de reducir el tiempo de preparación de sus alimentos.

- Segmentación conductual. La principal variable conductual por analizar es la asistencia a supermercados por parte de los NSE A y $\mathrm{B}$, lo cual representa el principal punto de venta potencial. Como se observa en la figura 2, casi la totalidad de amas de casa de dichos segmentos socioeconómicos asisten a los supermercados por lo menos una vez al mes.

Figura 2

Frecuencia de asistencia a supermercados por nivel socioeconómico (\%)

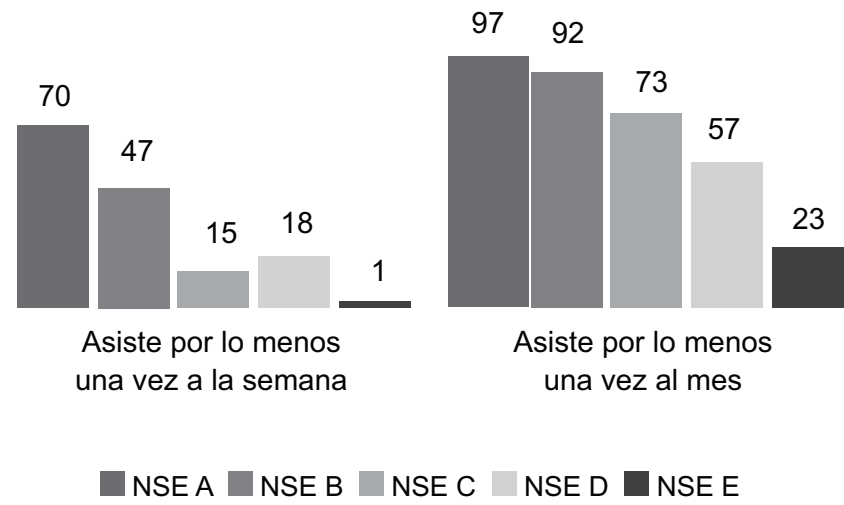

Base: Total de amas de casa entrevistadas (595).

Fuente: Ipsos Apoyo (2010).

Finalmente se escoge como mercado meta a los hogares de Lima metropolitana pertenecientes a los NSE A y B, que tengan el hábito de comprar puré de papa instantáneo para consumo en sus hogares. 


\subsection{Determinación de la demanda}

Para determinar la demanda del producto del presente estudio, primero se analizará la demanda del principal producto sustituto, el puré de papa instantáneo, en el mercado meta seleccionado. Luego se aplicarán factores de corrección de intención e intensidad de compra para poder estimar la demanda para el puré instantáneo de zapallo.

En primer lugar se requiere una proyección de la población de Lima Metropolitana entre los años 2011 y 2016. Para ello se decidió tomar la proyección realizada por el INEI (2009).

En cuanto a la segmentación, se extrae del mismo estudio que en el año 2011 el $5.4 \%$ de la población de Lima metropolitana pertenecía al NSE A y el $15.5 \%$ al NSE B. Se mantendrán estos valores como constantes en los 6 años para obtener una proyección conservadora.

Por otro lado, se debe tener en cuenta que, según los informes de patrones de consumo de Ipsos Apoyo, en el año 2011 solo el $21 \%$ de la población del NSE A de Lima Metropolitana consumió el puré de papa instantáneo y en el caso del NSE B solo el $16 \%$. A pesar de que la tendencia actual es el incremento en esta clase de productos, de fácil preparación y nutritivos, se mantendrán estos valores constantes a lo largo de los años, para ser lo más conservadores posible.

Finalmente, el dato del consumo per capita se obtuvo de las encuestas de ENAHO. Este es de $0.5 \mathrm{~kg}$ de puré instantáneo de papa al año. Se considerará un crecimiento anual del $5 \%$.

Con toda esta información, se obtiene la demanda proyectada para el puré instantáneo de papa para el mercado meta, que se muestra en la tabla 3. 


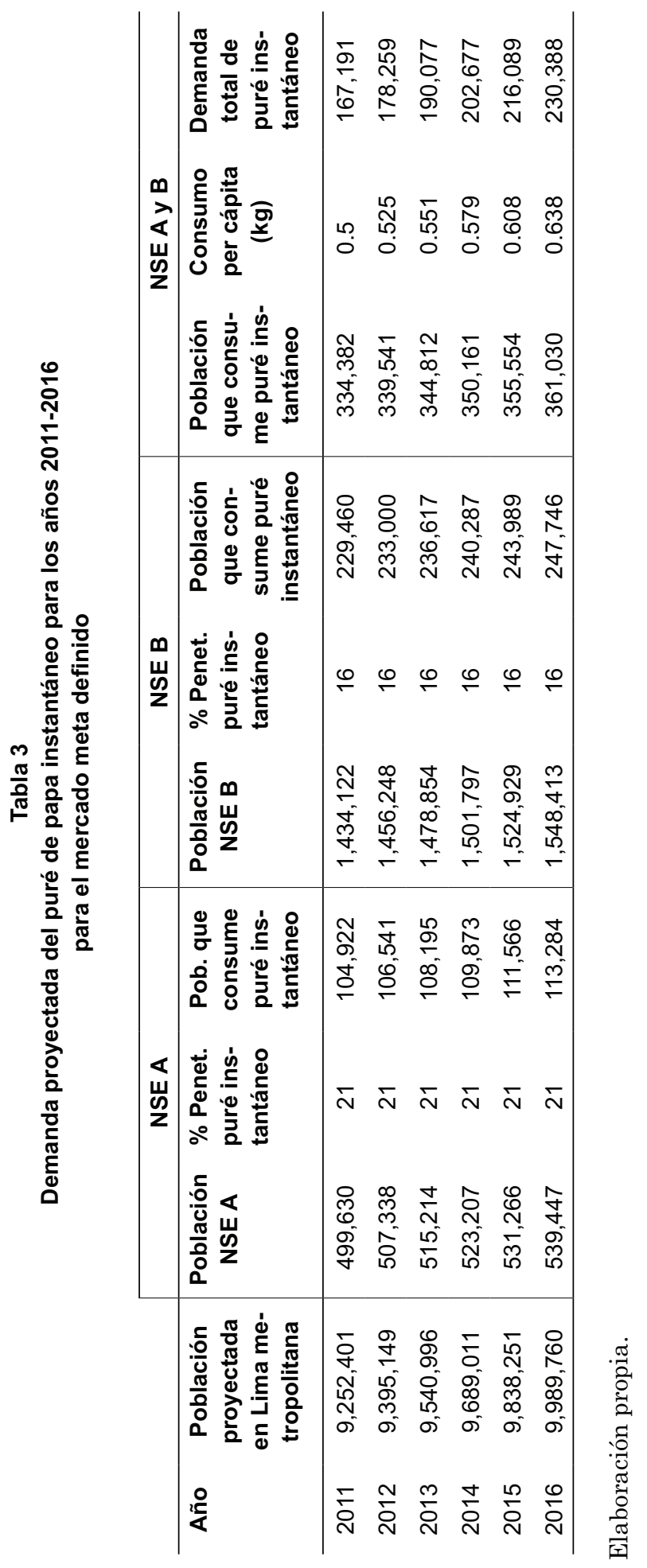




\subsection{Determinación de la demanda para el proyecto}

Para determinar la demanda del proyecto se partirá de la demanda hallada en la tabla 3. Como se mencionó antes, se deben ajustar los números con factores de corrección obtenidos como resultado de una encuesta realizada a 52 amas de casa. Estos factores cuantifican la intención e intensidad de compra de nuestro producto específico, el puré de zapallo:

Intención de compra: 86.54 \% (45 de 52 indicaron que sí comprarían el producto nuevo).

Intensidad de compra: $56.22 \%$ (véase la tabla 4).

Tabla 4

Intensidad de compra: ¿con qué seguridad compraría el producto?

\begin{tabular}{lcccc}
\hline Descripción & $\begin{array}{c}\text { Intensidad } \\
\text { de compra }\end{array}$ & $\begin{array}{c}\text { Cantidad de } \\
\text { encuestados }\end{array}$ & $\begin{array}{c}\text { Porcentaje de } \\
\text { encuestados }\end{array}$ & $\begin{array}{c}\text { Ponderación } \\
\text { (\%) }\end{array}$ \\
\hline Muy poco probable & 1 & 2 & 4.44 & 0.44 \\
Poco probable & 3 & 4 & 8.89 & 2.67 \\
Probable & 5 & 19 & 42.22 & 21.11 \\
Bastante probable & 6 & 11 & 24.44 & 14.67 \\
Muy probable & 8 & 6 & 13.33 & 10.67 \\
Completamente seguro & 10 & 3 & 6.67 & 6.67 \\
\hline & Total & 45 & 100 & 56.22 \\
\cline { 2 - 5 }
\end{tabular}

Elaboración propia.

A continuación, en la tabla 5, se muestra la demanda del proyecto aplicando los ratios: 
Tabla 5

Demanda para el proyecto de los años 2011-2016

\begin{tabular}{cccccc}
\hline Año & $\begin{array}{c}\text { Demanda pro- } \\
\text { yectada puré } \\
\text { instantáneo } \\
\text { de papa (kg) }\end{array}$ & $\begin{array}{c}\text { Factor } \\
\text { intención } \\
\text { de compra } \\
\mathbf{( \% )}\end{array}$ & $\begin{array}{c}\text { Factor } \\
\text { intensidad } \\
\text { de compra } \\
\mathbf{( \% )}\end{array}$ & $\begin{array}{c}\text { Demanda } \\
\text { del proyecto } \\
\mathbf{( k g )}\end{array}$ & $\begin{array}{c}\text { Demanda del } \\
\text { proyecto } \\
\text { en sobres } \\
\mathbf{( 1 2 5 ~} \mathbf{~})\end{array}$ \\
\hline 2011 & 167,191 & 86.54 & 56.22 & 81,343 & 651 \\
2012 & 178,259 & 86.54 & 56.22 & 86,728 & 694 \\
2013 & 190,077 & 86.54 & 56.22 & 92,478 & 740 \\
2014 & 202,677 & 86.54 & 56.22 & 98,608 & 789 \\
2015 & 216,089 & 86.54 & 56.22 & 105,133 & 842 \\
2016 & 230,388 & 86.54 & 56.22 & 112,090 & 897 \\
\hline
\end{tabular}

Elaboración propia.

\section{LOCALIZACIÓN DE LA PLANTA}

Para determinar la ubicación de la planta hay 6 factores importantes que deben evaluarse:

- Factor A: Disponibilidad de las materias primas

- Factor B: Cercanía al mercado

- Factor C: Disponibilidad de mano de obra

- Factor D: Servicios de agua y luz

- Factor E: Vías de transporte

- Factor F: Disponibilidad de terrenos

De estos, los 2 más importantes son el A y B, como se muestra en la tabla 6. 
Tabla 6

Tabla de enfrentamiento de factores para la localización

\begin{tabular}{|c|c|c|c|c|c|c|c|c|}
\hline & A & B & C & D & E & F & Puntaje & Ponderación (\%) \\
\hline A & 1 & 1 & 1 & 1 & 1 & 1 & 6 & $\mathbf{2 8 . 5 7}$ \\
\hline B & 0 & 1 & 1 & 1 & 1 & 1 & 5 & $\mathbf{2 3 . 8 1}$ \\
\hline C & 0 & 0 & 1 & 1 & 0 & 0 & 2 & $\mathbf{9 . 5 2}$ \\
\hline D & 0 & 0 & 0 & 1 & 1 & 1 & 3 & $\mathbf{1 4 . 2 9}$ \\
\hline E & 0 & 0 & 1 & 0 & 1 & 0 & 2 & $\mathbf{9 . 5 2}$ \\
\hline F & 0 & 0 & 1 & 0 & 1 & 1 & 3 & $\mathbf{1 4 . 2 9}$ \\
\cline { 5 - 8 }
\end{tabular}

Elaboración propia.

- Factor A: Disponibilidad de las materias primas

Uno de los factores fundamentales para la localización de la planta es la cercanía a la fuente de abastecimiento de su principal insumo, el zapallo macre. Este es un producto perecible y de un tamaño significativo, lo cual implica ciertas dificultades logísticas para su transporte. La proximidad de las materias primas a la planta garantiza bajos costos de transporte y cortos tiempos de su entrega.

A continuación (tabla 7) se presentan los tres departamentos con mayor producción de esta hortaliza.

Tabla 7

Producción de los tres departamentos con la mayor participación nacional de 2009

\begin{tabular}{lc}
\hline Departamento & Producción $(\mathbf{t})$ \\
\hline Ica & 37,751 \\
Arequipa & 35,830 \\
Lima & 30,137 \\
\hline
\end{tabular}

Fuente: INEI (2010). Perú: compendio estadístico. 
Es importante recalcar que la cantidad del insumo requerido para el proyecto puede ser suplido por cualquiera de las alternativas mencionadas, lo que es un buen motivo para localizar la planta en cualquiera de estos tres departamentos.

- Factor B: Cercanía al mercado

Seguidamente, para evaluar la cercanía al mercado, se compararán en la tabla 8 las distancias de los departamentos de Lima, Ica y Arequipa con respecto al mercado meta que se encuentra en Lima.

Tabla 8

Distancia de departamentos del Perú con respecto a Lima

\begin{tabular}{lc}
\hline Distancia $(\mathbf{k m})$ & Lima \\
\hline Arequipa & 966,89 \\
Ica & 303,21 \\
Lima & 0 \\
\hline
\end{tabular}

Fuente: MTC. Itinerario de rutas DGC-MTC.

Elaboración propia.

El mercado objetivo son los sectores A y B de Lima metropolitana, por lo que la alternativa más acertada sería instalar la planta en la ciudad de Lima, posiblemente en algún parque industrial.

\subsection{Evaluación y selección}

El siguiente paso para la selección de la localización es calificar cada una de las alternativas según la información que se tiene con respecto a cada factor. Se utilizará la matriz de calificación de factores mostrada en la tabla 9 . 
Tabla 9

Matriz de calificación de factores

\begin{tabular}{cl}
\hline Puntaje & \multicolumn{1}{c}{ Sustento } \\
\hline 2 & Malo: No cumple con ninguna expectativa del factor. \\
4 & Regular: Cumple con el mínimo de expectativas. \\
6 & Bueno: Cumple con las expectativas pero no las supera. \\
8 & Muy bueno: Supera las expectativas del factor. \\
10 & Excelente: Tiene las características ideales. \\
\hline
\end{tabular}

Elaboración propia.

Seguidamente, se procederá a realizar en la tabla 10 un ranking de factores, teniendo en cuenta la calificación de estos en cada departamento.

Tabla 10

Ranking de factores para la localización

\begin{tabular}{cccc|cc|cc} 
& & \multicolumn{2}{c|}{ Arequipa } & \multicolumn{2}{c|}{ Ica } & \multicolumn{2}{c}{ Lima } \\
\hline Factores & Ponderación & Calificación & Puntaje & Calificación & Puntaje & Calificación & Puntaje \\
\hline A & 28.57 & 8 & 229 & 10 & 286 & 8 & 229 \\
B & 23.81 & 6 & 143 & 8 & 190 & 10 & 238 \\
C & 9.52 & 6 & 57 & 6 & 57 & 8 & 76 \\
D & 14.29 & 8 & 114 & 6 & 86 & 10 & 143 \\
E & 9.52 & 8 & 76 & 8 & 76 & 10 & 95 \\
F & 14.29 & 6 & 86 & 6 & 86 & 8 & 114 \\
\hline & & Total & $\mathbf{7 0 5}$ & Total & $\mathbf{7 8 1}$ & Total & $\mathbf{8 9 5}$ \\
\hline
\end{tabular}

Elaboración propia.

Después de evaluar las diferentes alternativas de localización mediante el método de ranking de factores, se llegó a la conclusión de que el departamento indicado para la instalación de la planta de puré instantáneo de zapallo macre debe ser Lima. 


\section{INGENIERIIA DEL PROYECTO}

\subsection{Características del producto}

El puré de zapallo instantáneo consiste en un producto semielaborado a base de polvo de zapallo macre deshidratado, que puede ser reconstituido instantáneamente mediante la adición de agua hervida tibia. La dilución recomendada antes de consumirlo es de $90 \mathrm{~g}$ de puré en 240 a $300 \mathrm{ml}$ de agua hervida, dependiendo de la consistencia final preferida.

El producto será presentado en un empaque de doble laminado, uno exterior de plástico y uno interior de aluminio, con una capacidad de 125 g. Entre los principales insumos que se utilizarán se pueden destacar:

- Zapallo macre, como materia prima principal,

- Betacaroteno,

- Mix multivitamínico mineral (vitaminas A, B y C, calcio y hierro),

- Bolsas bilaminadas.

\subsection{Procesos de producción}

Existen diversos procesos para la producción de alimentos cocidos de reconstitución instantánea, específicamente purés.

El proceso de producción es el siguiente:

a) Recepción y selección de la materia prima

Se inicia con el arribo de un camión que trae la carga consolidada de zapallos necesaria para la producción diaria. La mercadería proviene del mercado mayorista de Santa Anita (antiguo mercado La Parada) de proveedores confiables que nos garantizan que el número de zapallos defectuosos hallados en planta sea bajo.

El camión se ubica en el muelle de descarga para iniciar el retiro de los zapallos. Inmediatamente se realiza una selección al $100 \%$ de los zapallos, eliminando aquellos que no estén en buen estado: con magulladuras, picaduras de insectos, brotes, hongos y golpes. Los zapallos en buen estado se trasladan al almacén, el cual cuenta con un sistema de refrigeración que permite la conservación óptima de este insumo a una temperatura entre los 10 y $12^{\circ} \mathrm{C}$. Los zapallos se colocan sobre plástico. 
b) Pesado de MP

Para iniciar el proceso de producción, primero se pesan los zapallos dentro del almacén para retirar únicamente la cantidad de kilogramos necesarios para la producción diaria. A su vez, se verifica visualmente que los zapallos escogidos estén lo suficientemente maduros. Los zapallos se trasladan mediante montacargas manuales a la zona de lavado.

c) Lavado

Se colocan los zapallos sobre un piso a desnivel con sistema de drenaje y se emplea un sistema de chorro de agua a presión constante, que consta de una lavadora de alta presión con manguera que se alimenta de 2 tanques de agua. El proceso se realiza en 3 etapas:

- Primero se realiza un lavado de arrastre para remover a presión toda impureza presente sobre la cáscara. Se emplea el agua clorada almacenada en uno de los tanques.

- Luego se conecta el sistema a un tanque con agua potabilizada con hipoclorito de sodio (10 ml de solución de hipoclorito de sodio al $10 \%$ por cada 100 litros de agua). La temperatura del agua es ligeramente elevada para aumentar la solubilidad del líquido y de esta manera poder eliminar más contaminantes. De esta forma se elimina cualquier microorganismo que se encuentre sobre la cáscara.

- Finalmente se vuelve a realizar un lavado con agua clorada para remover cualquier resto de hipoclorito que quede sobre la cáscara y no se contamine la pulpa durante el cortado.

d) Cortado y despepitado

Debido al gran tamaño de los zapallos, estos deben ser cortados en 6 pedazos grandes antes de ser pelados. Esta etapa es realizada por operarios con la ayuda de cuchillos eléctricos en mesas de trabajo de acero inoxidable, al lado de la zona de lavado. Inmediatamente después se revisa el interior del zapallo separando aquellos que se encuentren en mal estado o presenten cuerpos extraños. Al mismo tiempo se procede a retirar las pepas existentes. Los pedazos de zapallo son cargados por operarios hacia la zona de pelado y trozado.

e) Pelado y trozado

Se procede al pelado manual de la cáscara del zapallo empleando un disco rotatorio especial para pelar zapallos. Seguidamente los 
operarios cortan la hortaliza en trozos más pequeños con la ayuda de una herramienta cortadora de posición fija sobre mesas de acero inoxidable. La recepción de trozos se hace a través de canastillas que se colocan en una estantería rodante. De esta forma se trasladan los trozos hacia la marmita.

f) Cocción

Los trozos de zapallo se cocinan en una marmita a vapor con agitador en lotes de $220 \mathrm{~kg}$ aproximadamente en 30 minutos, bajo un espacio hermético. Durante la cocción se pierde alrededor del 3\% del peso de la materia prima. Pasado el tiempo indicado, se obtiene una pasta homogénea que es volcada a una faja transportadora inclinada donde se enfría para su ingreso directo a la máquina deshidratadora.

\section{g) Deshidratado}

La pasta ingresa a un deshidratador de túnel por aire caliente, en donde se somete a una corriente de aire caliente que luego quedará saturada de la humedad del producto. Este proceso se hace a una temperatura de $70{ }^{\circ} \mathrm{C}$ durante aproximadamente 45 minutos. Esta forma de secado es la que determina la capacidad de instantanización del producto terminado. Se deben formar en la estructura del zapallo deshidratado seudovías que permitan la fácil entrada de agua al momento de la reconstitución.

La pasta de zapallo ingresa con una humedad de $85 \%$ y se obtienen láminas de zapallo con solo $10 \%$. Este bajo nivel de humedad permite obtener una actividad de agua reducida y así se mejora la conservación del producto. Al finalizar el deshidratado, se toman muestras de cada lote para verificar si existe algún microorganismo y si el nivel de humedad es el adecuado. De no serlo, se reprocesa el lote.

\section{h) Molienda}

El zapallo deshidratado en láminas se transporta a un molino para convertirlo finalmente en copos. La operación se realizará en un molino de impacto. A la salida de este, se cuenta con un tamiz de 200 micras, cuyo material retenido será reprocesado hasta cumplir con dicha granulometría. El resultado final serán finos copos de zapallo que se almacenan temporalmente en el silo acoplado al molino. En esta operación, se pierde cerca del $2 \%$ del producto. 
j) Mezcla y homogeneizado

Una vez que el silo está lleno, se ingresa ese lote al tanque mezclador y se adicionan betacarotenos en una proporción de $150 \mathrm{mg}$ por cada $\mathrm{kg}$ de copos de zapallo y también un mix multivitamínico mineral (vitamina A, B y C, calcio y hierro) en una proporción de $500 \mathrm{mg}$ por cada $\mathrm{kg}$ de copos. Estos insumos se adicionan con el fin de contrarrestar las pérdidas parciales de los beneficios naturales del zapallo durante el procesamiento, especialmente en los procesos térmicos. Se utilizará un tanque mezclador con agitación para obtener un producto homogéneo.

k) Envasado y codificado

La mezcla ingresa a la tolva de una empacadora automática vertical que llena las bolsas y las sella con aire caliente. La máquina está programada para dosificar la cantidad de $125 \mathrm{~g}$ por bolsa. Las bolsas son de un material bilaminado para evitar principalmente el ingreso de la humedad al producto, y los efectos degradantes a los que se vería expuesto a causa de la luz (oxidación de carotenos, que generan decoloración) o el calor (pérdida de algunas vitaminas).

A la salida, la misma máquina codifica cada bolsa con detalles como la fecha de vencimiento, el lote de producción y la hora y fecha de envasado.

l) Control de calidad final

Se realiza un control de calidad final, para ello se retiran algunas unidades del lote de puré de zapallo embolsado con el fin de llevarlas al laboratorio de calidad con el fin de revisar los aspectos sensoriales, $\mathrm{pH}$, humedad, homogeneidad de la mezcla, viscosidad, peso neto del contenido del sobre y realizar pruebas microbiológicas.

m) Encajado y almacenamiento del PT

Finalmente, el encajado se realiza manualmente, en cajas de cartón. En cada caja se colocan 64 sobres. Las dimensiones de la caja máster son 40x30x60 cm. Dichas cajas se colocan sobre parihuelas para su traslado al almacén de productos terminados, donde se conservan en un ambiente fresco y seco ( $19^{\circ} \mathrm{C}$ y HR $70 \%$, aproximadamente).

A continuación se presenta el diagrama de operaciones del proceso. 
Figura 3

Diagrama de operaciones del proceso de producción del puré instantáneo de zapallo macre

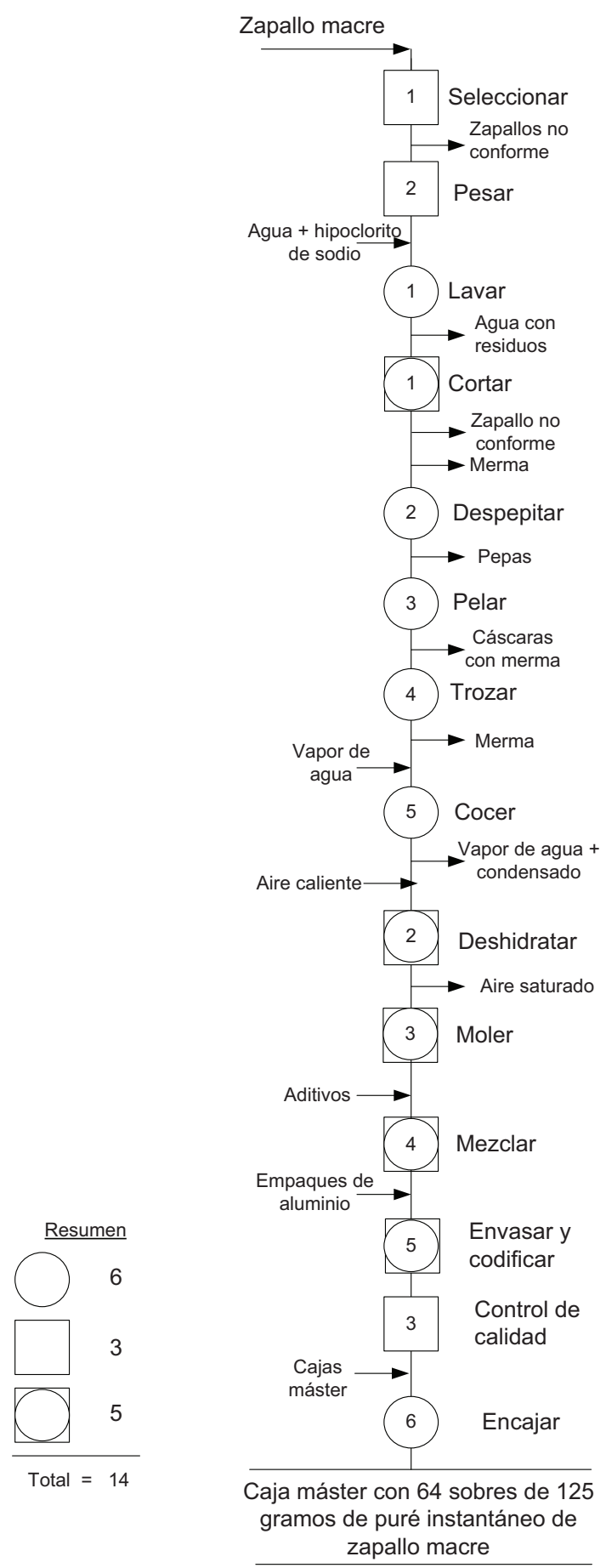

Elaboración propia. 


\section{INVERSIONES}

La inversión total estimada se presenta en la tabla 11.

Tabla 11

Inversión total estimada

\begin{tabular}{lr}
\hline Rubro & Monto (S/.) \\
\hline Maquinaria y equipos & 101,006 \\
Costo del terreno & 612,000 \\
Infraestructura del proyecto & 234,655 \\
Inversión fija intangible & 50,357 \\
Capital de trabajo & 187,272 \\
\hline Total & $\mathbf{1 , 1 8 5 , 4 9 0}$ \\
\hline
\end{tabular}

Elaboración propia.

En la tabla 12 se presenta la inversión que se realiza sobre aquellos activos constituidos por los servicios o derechos adquiridos necesarios para la puesta en marcha del proyecto; estos datos han sido tomados de un estudio similar (Doig, 2011).

Tabla 12

Inversión fija intangible

\begin{tabular}{lcc}
\hline Concepto & Costo (\$) & Costo (S/.) \\
\hline Estudio de factibilidad & 16,500 & 42,900 \\
Licencia de construcción & 190 & 494 \\
Marco legal & 678 & 1,763 \\
Gasto por capacitación del personal & 2,000 & 5,200 \\
\hline Total (S/.) & & $\mathbf{5 0 , 3 5 7}$ \\
\hline
\end{tabular}

Nota: Tasa de cambio del dólar: 2.55 soles.

Elaboración propia. 


\section{EVALUACIÓN ECONÓMICO-FINANCIERA}

\subsection{Evaluación económica}

Para la evaluación económica se considera que toda la inversión necesaria para el proyecto es aportada por los mismos accionistas. Por lo tanto, se evalúa bajo el costo de oportunidad del accionista (COK), el cual es igual al 18 \% y representa el riesgo que correría el accionista al invertir en este proyecto en lugar de otra mejor alternativa. Los indicadores del flujo de fondos son los siguientes:

VANE (18\%): S/.71,685

TIR: $20 \%$

B/C: S/. 1.06

PR: 64 meses (5 años y 4 meses)

\subsection{Evaluación financiera}

Para esta evaluación se considera el financiamiento externo como flujo de proyecto, incluidos los préstamos bancarios y, por lo tanto, el pago de intereses y las amortizaciones. La tasa que se va a utilizar es el costo promedio ponderado de capital (CPPC) que se halla con la siguiente fórmula: $\mathrm{CPPC}=\mathrm{TEA} *($ proporción financiamiento $)+\mathrm{COK}^{*}($ proporción capital propio).

$$
\mathrm{CPPC}=15.08 \% * 0.6+18 \% * 0.4=16.25 \% \text {. }
$$

A partir de esta tasa y del flujo de fondos financiero hallado se obtienen los siguientes indicadores:

VANF (16.25\%): S/. 244,937

TIR: $30 \%$

B/C: S/. 1.52

PR: 51 meses (4 años y 3 meses) 


\subsection{Análisis de los resultados económicos y financieros del proyecto}

Para realizar un análisis de resultados, se deben interpretar los 4 indicadores obtenidos en ambas evaluaciones:

1. El valor actual neto (VAN) es el valor resultante al traer todos los flujos netos al presente. Si el VAN es mayor que 0, el proyecto es rentable. En ambos casos se obtienen resultados positivos, siendo el VANF mayor que el VANE.

2. La tasa interna de retorno (TIR) es la tasa de descuento que hace que el VAN sea igual a 0 , en otras palabras, es aquella tasa que iguala la suma de los flujos netos traídos al presente a la inversión inicial. El criterio en este caso para determinar un proyecto rentable es si la TIR es mayor que la tasa de descuento utilizada en el flujo. $\mathrm{Al}$ igual que en análisis del VAN, ambas evaluaciones se proyectan como rentables, siendo el TIRF mayor que el TIRE.

3. La relación beneficio/costo (B/C) es un indicador que vincula los flujos netos actualizados con la inversión. Una interpretación sencilla consiste en tomar el número obtenido como el beneficio que se obtiene por cada sol invertido. El criterio para aceptar el proyecto sería si el B/C es mayor de 1. En ambas evaluaciones, se excede este número; la financiera es más positiva.

4. El periodo de recupero (PR) indica el tiempo en el cual se recuperaría la inversión, a la misma tasa de descuento del proyecto. Desde una métrica económica, si la inversión proviene únicamente de capital propio, se recuperaría en 5 años y 4 meses; bajo un enfoque financiero, si se accede a un préstamo bancario del $60 \%$ del total de la inversión, se recuperaría en 4 años y 3 meses.

Se concluye que para el accionista es más rentable acceder a un préstamo que financiarse por sí mismo.

\section{CONCLUSIONES Y RECOMENDACIONES}

1. El presente estudio concluye que la ejecución de un proyecto de instalación de una planta procesadora de puré instantáneo de zapallo macre es factible, ya que existe un mercado meta dispuesto a consumir el producto conformado por las amas de casa pertenecientes a los NSE A y B de Lima, cuyos patrones de consumo se ajustan a 
los beneficios que ofrece el producto propuesto. Se determinó una demanda de 650,745 sobres de $125 \mathrm{~g}$ para el primer año y se estableció un precio de venta de S/. 2.60.

2. Lima es el lugar apropiado para la ubicación de la planta, teniendo en cuenta factores como la disponibilidad de la materia prima y la cercanía al mercado meta.

3. La inversión requerida es de S/. 1,185,490, monto que será financiado de la siguiente manera: $40 \%$ por los accionistas y $60 \%$ por Cofide, con un préstamo de cuotas constantes por los próximos 6 años de operación.

4. La evaluación financiera presenta un VANF de S/. 244,937, así como una TIR del 30\%, motivo por el cual es posible corroborar la viabilidad del presente proyecto. De la misma manera, se realizó un análisis de sensibilidad, en el cual se consideran las variables precio, costo de la materia prima y la tasa de interés del préstamo; la primera es la que más afectaría la rentabilidad.

\section{REFERENCIAS}

Baca, R. (2007). Estudio preliminar para la instalación de una planta de envasados de mermelada y mazamorra de zapallo macre. Trabajo de investigación del Seminario II. Lima: Universidad de Lima.

Díaz, B., Jarufe, B., \& Noriega, M. (2007). Disposición de planta. Lima: Universidad de Lima, Fondo Editorial.

Doig Castillo, G. E. (2011). Estudio de prefactibilidad para la instalación de una planta fabricadora de snacks a partir de fritura de yacón. Trabajo de investigación para optar el título profesional de ingeniero industrial. Universidad de Lima, Lima.

Huanachín, W. (13 de agosto del 2012). Precio de terrenos industriales desalienta a empresas foráneas. Gestión. Lima.

Instituto Nacional de Estadística e Informática (INEI). (2009). Perú: estimaciones y proyecciones de población total y por sexo de las ciudades principales, 2000-2016. Boletín Especial 23. Recuperado de www.inei.gob.pe/biblioineipub/bancopub/Est/Lib1020/index. html 
Instituto Nacional de Estadística e Informática (INEI). (2010). Perú: compendio estadístico 2010. Recuperado de http://www.inei.gob. pe. Base de datos disponible en la Universidad de Lima.

Instituto Nacional de Estadísticas e Informática (INEI). (2012). Encuesta Nacional de Hogares (Enaho) 2011. Recuperado de www.inei.gob.pe/biblioineipub/bancopub/Est/Lib1020/index. html

Instituto Nacional de Defensa de la Competencia y de la Protección de la Propiedad Intelectual (Indecopi). (2004). NTP 209.260:2004, Revisión 2004: Alimentos cocidos de reconstitución instantánea.

Ipsos Apoyo. (2008). Informe gerencial de marketing: Liderazgo en productos alimenticios 2007. Marketing Data Plus. Recuperado de http://www.ipsos_apoyo.org.com.pe.

Ipsos Apoyo. (2010). Informe gerencial de marketing: Tendencias en salud y alimentación 2009. Marketing Data Plus. Recuperado de http://www.ipsos_apoyo.org.com.pe.

Ipsos Apoyo. (2011). Informe gerencial de marketing. Estadística poblacional 2011. Marketing Data Plus. Recuperado de http:// www.ipsos_apoyo.org.com.pe

Ipsos Apoyo. (2011). Informe gerencial de marketing. Perfil del ama de casa 2010. Marketing Data Plus. Recuperado de http://www. ipsos_apoyo.org.com.pe.

Ipsos Apoyo. (2011). Informe gerencial de marketing: Liderazgo en productos comestibles 2010. Marketing Data Plus. Recuperado de http://www.ipsos_apoyo.org.com.pe.

Lorello, I., Asprelli, P., Peralta, E., Makuch, M., \& García, S. (2007). Caracterización de zapallos "criollos" (Cuburbita maxima). Mendoza, Argentina.

Ministerio de Transportes y Comunicaciones. Itinerario de rutas DGCMTC. Recuperado de http://www.mtc.gob.pe

Moreiras, O., Carvajal, A., Cabrera, L., Cuadrado, M. (2001). Tablas de composición de alimentos. Madrid: Ediciones Pirámide.

Rodríguez, M. (2010). Estudio preliminar para la instalación de una planta procesadora de puré instantáneo de camote. Trabajo de investigación del Seminario II. Lima: Universidad de Lima. 